\title{
Imaging of Right-to-Left Shunt in an Adult Patient with Unroofed Coronary Sinus with Persistent Left Superior Vena Cava Diagnostic Clue and Pitfall
}

\author{
Chieko Sakai, ${ }^{1}$ MD, Tetsuhiro Yamano, ${ }^{1,2}$ MD, Tomonori Miki, ${ }^{1}$ MD, Akiko Otsuka, ${ }^{2}$ BS, \\ Yuzuko Kato, ${ }^{2}$ BS, Michiyo Yamano, ${ }^{1} \mathrm{MD}$, Takeshi Nakamura, ${ }^{1}$ MD and Satoaki Matoba, ${ }^{1} \mathrm{MD}$
}

\begin{abstract}
Summary
A 54-year-old woman with a history of multiple cardiac surgeries suffered from hypoxemia caused by a right-to-left intra-cardiac shunt due to coronary sinus (CS) anomaly with persistent left superior vena cava (PLSVC). Both the contrast echocardiography and enhanced computed tomography (CT) provided conclusive diagnosis of this rare congenital anomaly, which was overlooked for a long time. However, an important diagnostic clue was left-arm injection of the contrast media. In the present case, previously performed enhanced CT with its routine manner, i.e., contrast through the right arm, missed this anomaly. It is crucial to note that the unusual type of unroofed CS with PLSVC, presenting with an entirely right-to-left intra-cardiac shunt, cannot be delineated on an enhanced routine chest CT if the contrast media is injected through the right arm.
\end{abstract}

(Int Heart J 2017; 58: 1008-1011)

Key words: Echocardiography, Computed tomography, Diagnosis

$\mathrm{U}$ nroofed coronary sinus (CS) is a rare congenital cardiac anomaly with a reported prevalence of only $0.1 \%$ among all congenital heart diseases. ${ }^{1)}$ In this pathology, there is a significant communication between the CS and the left atrium (LA) due to complete or partial absence of the roof or septum between the CS and the LA. Other than persistent left superior vena cava (PLSVC), which is complicated with as much as $75 \%$ of the cases of this pathology, ${ }^{1)}$ other complicated malformations could critically affect the clinical manifestations of unroofed $\mathrm{CS}^{2}{ }^{2)}$ Here, we describe an adult case who suffered from hypoxemia due to a variant form of this pathology overlooked for a long time; then, we reemphasize "classical" diagnostic clues and pitfalls of this pathology that we learned from the present case.

\section{Case Report}

A 54-year-old woman was referred to our department for evaluation of hypoxemia. She had a history of multiple cardiac surgeries: repair of pulmonary artery stenosis during childhood, repair of ruptured sinus of Valsalva at 25 years of age, and aortic valve replacement at 35 years of age. She underwent aortic valve re-replacement for prosthetic valve regurgitation 13 months ago. She had taken medications for hypertension after the latest surgery. Although hypoxemia was noted before she underwent the latest cardiac surgery, the etiology was considered as transient pulmonary congestion caused by a prosthetic valve regurgitation. Following successful surgery, arterial oxygen saturation of less than $90 \%$ at rest on room air was finally recognized as hypoxemia of unknown etiology. Her physical examination was unremarkable, without grade $2 / 6$ systolic ejection murmur heard at the aortic area. A chest radiogram did not show pulmonary congestion. Transthoracic echocardiography revealed abnormal ventricular septal motion, presumably due to postoperative pericardial adhesions. However, there was neither evidence of elevated left ventricular filling pressure nor abnormalities in the postsurgical lesions. Moreover, the CS did not appear dilated on the transthoracic echocardiography. Before the latest surgery, coronary computed tomography (CT) angiography had been performed (Figure 1). As per the standard protocol for CT angiography, ${ }^{3)}$ a contrast agent had been administered through the right arm. There were no significant findings other than the implanted prosthetic mechanical aortic valve and a PLSVC (Figure 1A-C, arrows). Contrast transesophageal echocardiography using agitated saline was performed to detect the intra-cardiac shunt. To evaluate the right-to-left shunt through the patent foramen ovale, we injected saline through the right arm: A moderate amount of the contrast agent was found in the LA, across the patent foramen ovale. Furthermore, we injected saline through the left arm to examine the un-

From the 'Department of Cardiovascular Medicine, Graduate School of Medical Science, Kyoto Prefectural University of Medicine, Kyoto, Japan and ${ }^{2}$ Department of Laboratory Medicine, Graduate School of Medical Science, Kyoto Prefectural University of Medicine, Kyoto, Japan.

Address for correspondence: Tetsuhiro Yamano, MD, Department of Cardiovascular Medicine/Laboratory Medicine, Graduate School of Medical Science, Kyoto Prefectural University of Medicine, 465 Kajii-cho, Kamigyo-Ku, Kyoto 602-8566, Japan. E-mail: tyamano@koto.kpu-m.ac.jp or teyamano@ hotmail.co.j $\mathrm{p}$

Received for publication October 14, 2016. Revised and accepted January 13, 2017.

Released in advance online on J-STAGE November 17, 2017.

doi: 10.1536/ihj.16-517

All rights reserved by the International Heart Journal Association. 

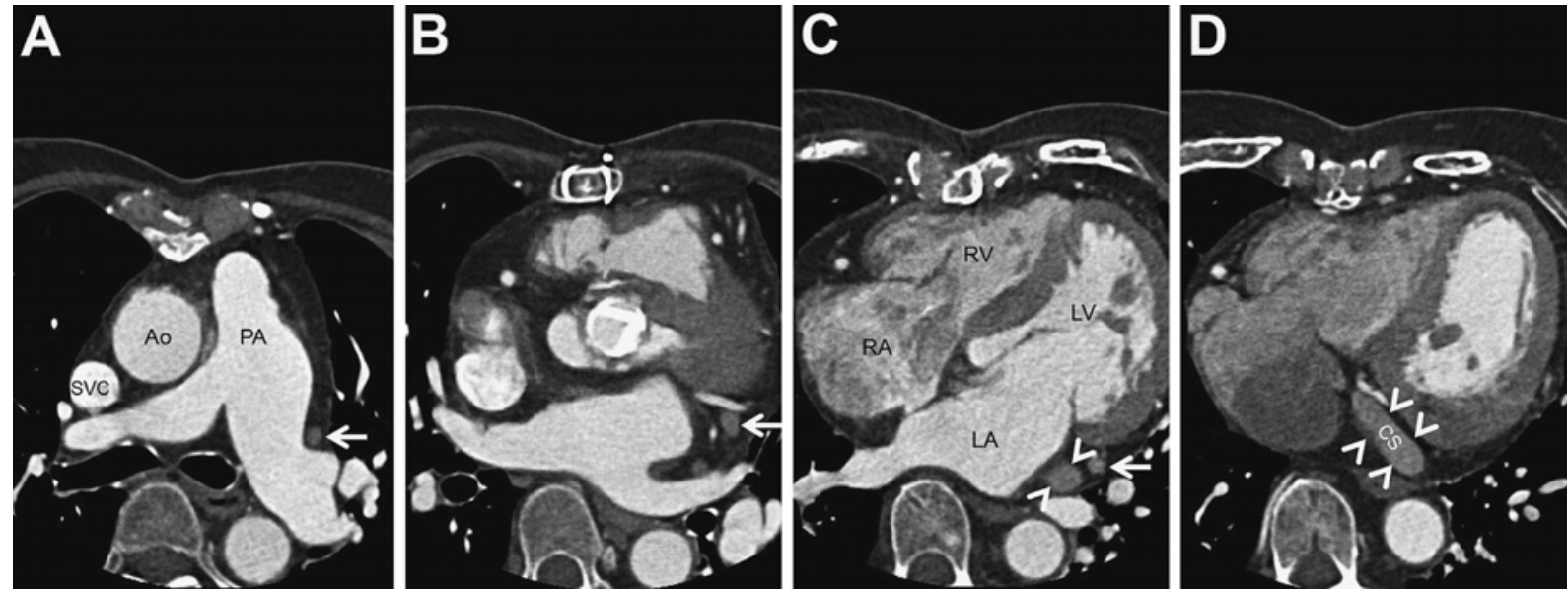

Figure 1. Contrast-enhanced 64-row multi-detector computed tomography (CT; Brilliance 64, Philips Healthcare, Best, The Netherlands) was performed before aortic valve re-replacement for the purpose of coronary CT angiograms. Although it was not fulfilled by contrast medium because contrast was injected through the right arm according to the usual manner of CT angiograms, it could be diagnosed that persistent left superior vena cava (PLSVC; A-C, arrows) drained into the coronary sinus (CS; C, D, arrowheads). However, the other structural intra-cardiac abnormality was not obvious. Ao indicates aorta; LA, left atrium; LV, left ventricle; PA, pulmonary artery; RA, right atrium; RV, right ventricle; and SVC, superior vena cava.
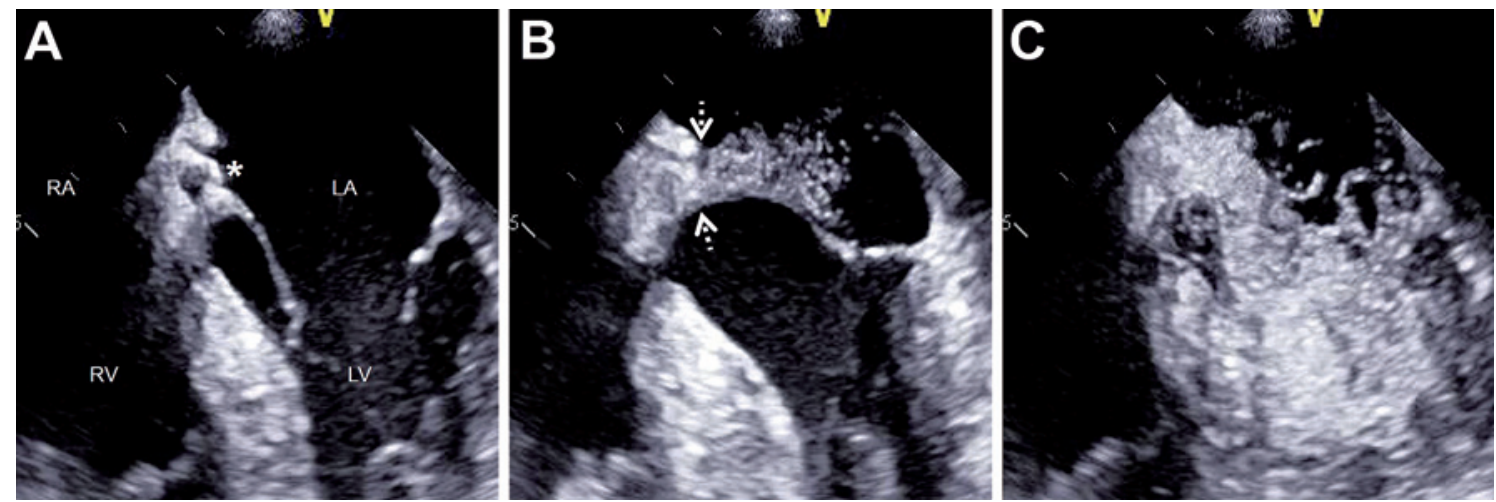

Figure 2. Contrast transesophageal echocardiograms. Agitated saline injected through the left arm, mostly appeared in the LA (B, dotted arrows) through a curious opening in the atrioventricular crux (A, asterisk). $\mathbf{A}$ to $\mathbf{C}$ are serial images. Abbreviations as in Figure 1.

roofing of CS connected with PLSVC (Figure 2A-C, are serial images). Surprisingly, the majority of the contrast agent injected through the left arm entered the LA (Figure $2 \mathrm{~B}$, dotted arrows) from a curious opening in the crux (Figure 2A, asterisk) connecting with the CS. The contrast agent did not appear in the right heart chambers through the CS, suggesting the diagnosis of a variant type of unroofed $\mathrm{CS}$, with atresia of the $\mathrm{CS}$ orifice to the right atrium (RA). ${ }^{2,4}$ Based on these results, enhanced CT images were again obtained by injecting contrast agent through the left arm (Figure 3). First-pass images demonstrated that PLSVC (Figure 3A-C, arrows) drained into CS (Figure 3C, D, arrowheads), and the majority of the contrast agent finally appeared in the LA (Figure 3C, dotted arrows), confirming our echocardiographic diagnosis. The angiograms and corresponding 3-dimensional CT images are shown (Figure $4 \mathrm{~A}-\mathrm{C}$, respectively; and Supplemental Figure). Since the left innominate vein (LIV) was patent (Figure 4C, open arrow), we could easily perform a percutaneous transcatheter PLSVC embolization, using
Amplatzer $^{\mathrm{TM}}$ Vascular Plug II (St. Jude Medical, Minneapolis, MN, USA) (Figure 4D). The patient's hypoxemia improved with arterial oxygen saturation of $94 \%$ on room air, and a CT performed three months after the procedure showed a PLSVC occlusion (Figure 4E); thereafter, she has been carefully followed up.

\section{Discussion}

In the present case, the PLSVC on the previous CT indicated injection of agitated saline through the left arm for contrast echocardiography, which was a definitive clue to the correct diagnosis of the unroofed CS. Generally, intra-cardiac shunt flow in patients with unroofed CS is expected to be in left-to-right direction; however, its amounts and directions, i.e., either left-to-right or right-toleft, can vary depending on the communication size, the inter-atrial pressure gradient, and complicated malformations. ${ }^{2,5)}$ In our patient, there was a curious vascular channel connecting the CS and the LA. This was considered as 

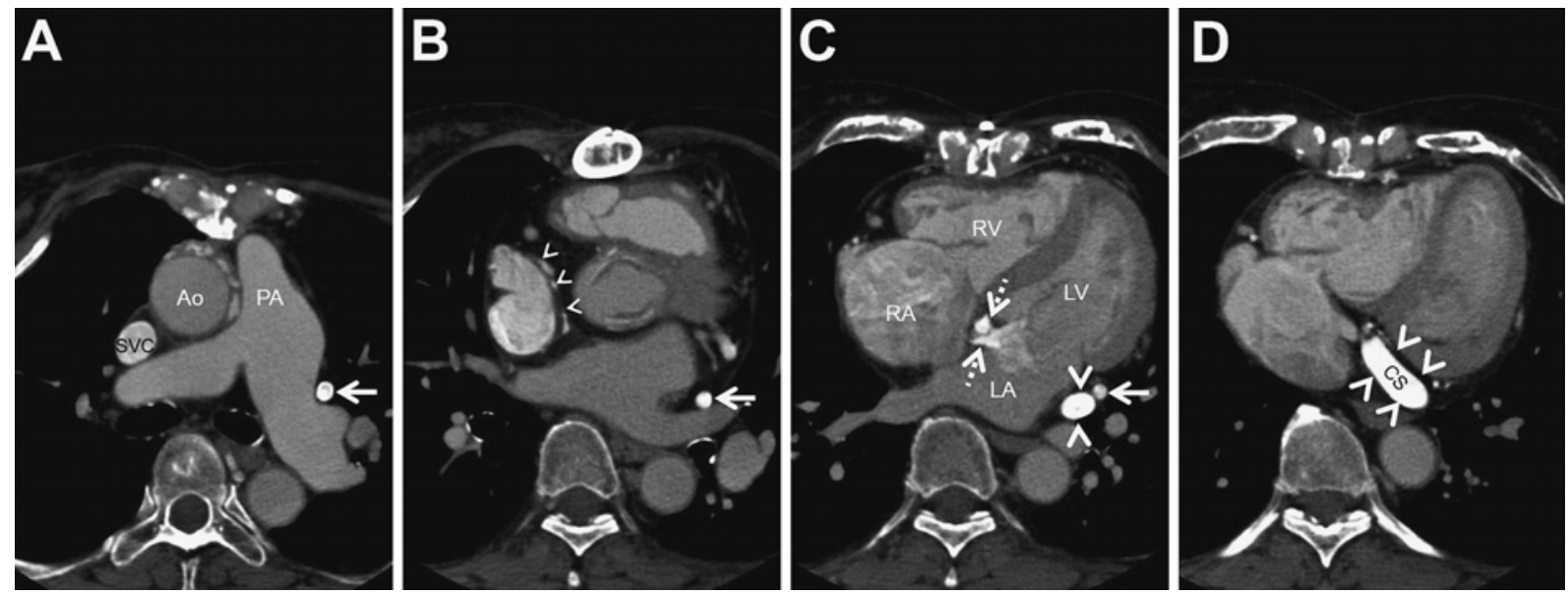

Figure 3. Contrast-enhanced 320-row multi-detector CT (Aquilion ONE, Toshiba Medical Systems, Tokyo, Japan) was again performed by injecting contrast through the left arm. In the first-pass images, the PLSVC (A-C, arrows) was well enhanced, and the majority of contrast appeared in the LA (C, dotted arrows) through the CS (C, D, arrowheads). Other than innominate vein followed by SVC, there were some abnormal vascular channels through which a small amount of contrast appeared in RA and RV (B, small arrowheads). Abbreviations as in Figure 1.
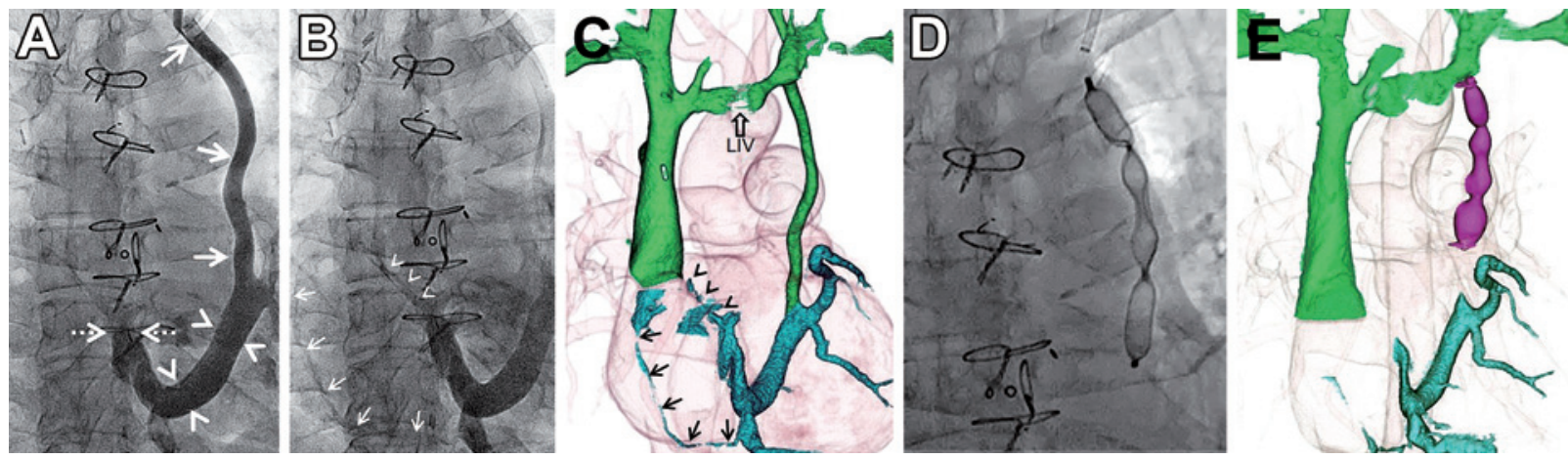

Figure 4. A: Selective angiography of the PLSVC (arrows) well delineated an overview of this abnormal circulation. Arrowheads indicate CS and dotted arrows, CS opening to the LA. B: Delayed image showed a small amount of contrast coming into RA through the collateral vessel connecting to the small cardiac vein (B, C, small arrows) and small vascular channel between CS and RA (B, C, small arrowheads). C: Corresponding 3-dimensional CT image obtained at a delayed equilibrium phase. The existence of left innominate vein (LIV, open arrow) was an additional important finding to the angiograms. D: Fluoroscopy when performing percutaneous transcatheter PLSVC embolization. E: Three-dimensional CT image after the embolization revealed PLSVC occlusion. Other abbreviations as in Figure 1.

an atypical type of unroofed $\mathrm{CS} .^{4)}$ Moreover, she was considered as having atresia of CS orifice, which is also a very rare cardiac malformation, with a reported incidence rate of $0.03 \%$ in the general population ${ }^{6,7)}$ In the literature ${ }^{8)}$ reviewing 37 previously described cases with atresia of CS orifice, unroofed CS was complicated in 9 (24\%). In the present case, as a result of an unroofed CS complicated with both PLSVC and atresia of CS orifice, venous flow from PLSVC, as well as the coronary vein, drained into the LA, presenting entirely right-to-left intra-cardiac shunt similarly to the previously reported case. ${ }^{5}$

Owing to the recent widespread use of coronary CT angiography, ${ }^{9,10)}$ there are some reports describing the incidental identification of unroofed CS in asymptomatic patients on CT imaging. ${ }^{11,12)}$ However, it should be kept in mind that the usual timing of CT image acquisition is set to obtain the maximum enhancement of arterial structure. Therefore, when the contrast media is injected through the right arm in patients with unroofed CS and PLSVC, if there is no left-to-right shunt through an unroofed CS, the contrast enhancement cannot be observed in the CS. Hence, in our case, the preoperative coronary CT angiography missed the unroofed $\mathrm{CS}$; thus, significant hypoxemia remained after the latest cardiac surgery.

The contrast echocardiography has been reported as a useful method for diagnosing various types of unroofed $\mathrm{CS} ;{ }^{1)}$ moreover, it is more valuable in patients with unroofed CS complicated with PLSVC than in those without PLSVC. On echocardiograms, PLSVC is often suspected in the presence of CS dilatation. However, the CS dilatation in the present case was not significant $(10 \mathrm{~mm}$ on transesophageal echocardiograms and $11 \mathrm{~mm}$ on the CT images ${ }^{4,6)}$ ). It might reflect comparatively small amounts of systemic venous blood returning to the LA via PLSVC with a calculated right-to-left shunt fraction of $11 \%$, since LIV was patent as the other venous route to the RA and PLSVC finally drained to the LA, of which the chamber pressure was equivalent to that of RA in the present case 
(Supplemental Table). Subsequent to the echocardiographic diagnosis of PLSVC draining into the LA via the unroofed CS, we performed another CT, injecting the contrast media through the left arm. In addition to the confirmation of the echocardiographic diagnosis, the CT images demonstrated the bridging innominate vein, which was clinically valuable information in deciding treatment.

It is not clarified why her hypoxemia became prominent during middle age. Judging from pre-procedural normal intra-cardiac pressure (Supplemental Table), right heart failure that could exacerbate right-to-left shunt, was not concerned. Thus, we cannot entirely deny that her intra-cardiac shunt flow was iatrogenically caused with retrograde cardioplegia or LA venting at the previous surgeries. However, both details of initial three surgeries and the exact progress of her hypoxemia were not identified since these surgeries were performed more than 20 years ago, and she had been followed up in the other institution for a long time. At the latest surgery, although vent was placed in the LA, retrograde cardioplegia could not be performed since severe cardiac adhesion precluded RA incisions. Furthermore, she had suffered from hypoxemia preceding the latest surgery, suggesting that her intracardiac shunt had existed at least before the latest surgery.

In conclusion, in patients who are clinically suspected to have right-to-left shunts, i.e., systemic desaturation or brain abscess, and who have been already known or assumed to have PLSVC; we should differentiate unroofed CS of various types. Although both contrast echocardiography and CT imaging are valuable for diagnosis of this pathology, it is important to inject the contrast agent through the left arm. It is crucial to note that the unusual type of unroofed CS with PLSVC, presenting with an entirely right-to-left intra-cardiac shunt as in the present case, cannot be clearly delineated on an enhanced routine chest $\mathrm{CT}$ if the contrast media is injected through the right arm.

\section{Disclosures}

Conflicts of interest: None declared.

\section{References}

1. Xie MX, Yang YL, Cheng TO, et al. Coronary sinus septal de- fect (unroofed coronary sinus): echocardiographic diagnosis and surgical treatment. Int J Cardiol 2013; 168: 1258-63.

2. Mantini E, Grondin CM, Lillehei CW, Edwards JE. Congenital anomalies involving the coronary sinus. Circulation 1966; 33: $317-27$.

3. Abbara S, Blanke P, Maroules CD, et al. SCCT guidelines for the performance and acquisition of coronary computed tomographic angiography: A report of the society of Cardiovascular Computed Tomography Guidelines Committee: Endorsed by the North American Society for Cardiovascular Imaging (NASCI). J Cardiovasc Comput Tomogr 2016; 10: 435-49.

4. Kim H, Choe YH, Park SW, et al. Partially unroofed coronary sinus: MDCT and MRI findings. AJR Am J Roentgenol 2010; 195: W331-6.

5. Shirakawa K, Kawamura A, Muraoka N, et al. Positional desaturation due to persistent left superior vena cava draining into the left atrium. Heart Vessels 2016; 31: 828-30.

6. Song G, Ren W, Chen Y. Coronary sinus orifice atresia associated with persistent left superior vena cava: a case report with literature review. Echocardiography 2016; 33: 926-31.

7. Okuyama Y, Oka T, Mizuno H, Sakai T, Hirayama A, Kodama K. A case of atrioventricular nodal reentrant tachycardia with atresia of the coronary sinus ostium. Int Heart J 2005; 46: 899902.

8. Watson GH. Atresia of the coronary sinus orifice. Pediatr Cardiol 1985; 6: 99-101.

9. Koga S, Ikeda S, Nakata T, Maemura K. Spontaneous spiral dissection of left internal thoracic artery graft. Int Heart J 2015; 56: $360-2$

10. Niwa R, Hasumi E, Fujiu K, et al. A case of multiple coronary artery-left ventricular micro fistulae complicated with hepatic arteriovenous fistulae. Int Heart J 2016; 57: 123-6.

11. Thangaroopan M, Truong QA, Kalra MK, Yared K, Abbara S. Images in cardiovascular medicine. Rare case of an unroofed coronary sinus: diagnosis by multidetector computed tomography. Circulation 2009; 119: e518-20.

12. Kim HR, Yoo SM, Lee HY, et al. A Case of complete unroofed coronary sinus syndrome combined with coronary sinus stenosis leading to asymptomatic presentation. Iran J Radiol 2015; 12: e 16063

\section{Supplemental Files}

Supplemental Table

Supplemental Figure

Please see supplemental files;

https://www.jstage.jst.co.jp/article/ihj/58/6/58_16-517/_article/supple ment 